\title{
Diseño y simulación de un amplificador "Instrumental Operacional" como apoyo en la lectura y transmisión de señales mioeléctricas por un solo canal con bajo ruido
}

\author{
Design and simulation of an amplifier "Instrumental \\ Operational" to support reading and transmission of \\ myoelectric signals over a single channel with low noise
}

\author{
E. D. Aguirre Mesa ${ }^{1,2}$, D. A. García Arango ${ }^{1,3}$, C. F. Henao Villa ${ }^{1,4}$
}

Recibido: 20 de octubre 2016

Aceptado: 15 de abril 2017

\section{Resumen}

La importancia de diseñar un AIO (Amplificador Operacional Instrumental), es la necesidad de un desarrollo y producción de elementos electrónicos que ayuden a la interface mioelectriaca, en la que sin depender de fuentes de poder o energía altas, se pueda controlar dispositivos con los movimientos básicos del ser humano, a través de alguno de sus miembros articulados, para esto se requiere que tenga incorporado un amplificador operacional - instrumental. La más utilizada a la fecha son las de dos etapas, compuesta por un diferencial de entrada y un inversor de salida, estas, aun no cuentan con etapas de limpieza, filtro, frecuencia y longitud de la señal para ser tipificadas para ciertas operaciones y acciones básicas con especial atención a los aspectos de diseño que minimizan el ruido y la tensión del amplificador.

\footnotetext{
1 Corporación Universitaria Americana

2 eaguirre@americana.edu.co

3 dagarcia@coruniamericana.edu.co

4 chenao@coruniamericana.edu.co
} 
Aguirre, et al. / Diseño y simulación de un amplificador "Instrumental Operacional"...

La investigación inicia con un modelado y diseño de los componentes de filtros y reducción de ruidos con AIO, que capturan la señal por un canal, para poder ir escalando en complejidad y operabilidad, todo esto apoyado en un análisis experimental y soportado por el marco CDIO (Concepcion, Diseño, Implementación y Operatividad). Se desarrolla un primer modelo lógico de un prototipo manipulado con músculo, este sirve de plataforma para el movimiento de un servomotor y controlar como minimo 2 posibles movimientos. En trabajos futuros éstos serán documentados y gestionados a través de un software que valide la reducción significativa de ruido. Se deberá adquirir una mayor comprensión y conocimiento del ruido, la Interferencia electromagnética o EMI y sus causas ya que puede ayudar en el paso posterior de la cadena de procesado, que es su eliminación o disipación, por ello se tiene previsto asignar un tiempo al mejor conocimiento del ruido y al estudio de los sistemas de adquisición orientados a su reducción.

Palabras clave: Amplificador Operacional Instrumental, Señales Mioelectricas, Ruido Electromagnético, Mono canal

\section{Abstract}

The importance of designing an AIO (Operational Amplifier Instrumental), is the need for development and production of electronic elements that help mioelectriaca interface, which without relying on sources of power or high power, you can control devices with the basic movements of human beings, through one of its articulated members, for this is required to have built an operational amplifier - instrumental. The most commonly used to date are two stages, comprising a differential input and an inverter output, they even do not have cleaning steps, filter, frequency and length of the signal to be criminalized for certain operations and actions basic with focus on design aspects that minimize noise and voltage amplifier.

The investigation started with a modeling and design of components filters and noise reduction with $\mathrm{AIO}$, capturing the signal on a channel, to go climbing in complexity and operability, all supported by an experimental analysis and supported by the frame CDIO (conception, design, implementation and operation). a first logical model of a manipulated with some muscle prototype is developed, this provides a platform for the movement of a servomotor and controlling at least 2 possible moves, in future work they will be documented and managed through a software that validates the significant reduction noise. It must acquire a greater understanding and knowledge of noise, electromagnetic interference, or EMI and its causes as it can help in the later stage of the processing chain, which is the removal or dissipation, so it plans to allocate time to better noise knowledge and study of procurement systems aimed at their reduction..

Keywords: Operational Amplifier Instrumental, myoelectric signals, electromagnetic noise, Monocanal 


\section{Introducción}

Ly en día el desarrollo de Amplificadores Operacionales Instrumentales - AlO va en ascenso, pero son de muy alto costo, difícil desarrollo e implementación, ya que para su elaboración y construcción se está utilizando tecnología cerrada o patentada y su uso ya no solo se basa específicamente en el desarrollo de aplicaciones terapéuticas y de rehabilitación, milicia, industria y demás ${ }^{[1,[2]}$.

Este trabajo presenta el diseño y construcción de un sistema para la adquisición de señales electromiográficas provenientes de los músculos bíceps y tríceps, así como la metodología seguida para obtener los resultados obtenidos. La finalidad del trabajo consiste en plantear la primera etapa para la construcción de un sistema de lectura y transmisión de señales mioeléctricas que pueda reconocer los potenciales de acción generados por los músculos y mediante un algoritmo de aprendizaje como el de las redes neuronales o un software de toma de decisiones finita TDF, y el cual sea capaz de reconocer los potenciales de disparo de Electromiografía y catalogarlos como alguno de los movimientos del brazo o articulación involucrada.

Una de las topologías más utilizadas para la implementación de amplificadores operacionales CMOS es la de dos etapas, compuesta por un diferencial de entrada y un inversor de salida [3]. Usualmente se emplea un capacitor de Miller para efectuar la compensación en frecuencia, razón por la que el circuito es referido como operacional de Miller.

El mismo presenta, cuando es adecuadamente diseñado, un desempeño similar a topologías más modernas. Adicionalmente puede utilizarse para manejar cargas resistivas mediante el agregado de una etapa buffer de salida, lo anterior es tratado en el documento de los autores Laker and Sansen ${ }^{[1]}$. Teniendo presente que se debe tratar varios filtros que participen en la reducción de ruido electromagnético y por ende incorporar etapas de pre y posfiltrado al amplificador Operacional Instrumental [4],[5].

Se aborda en este trabajo el desarrollo y simulación de un amplificador operacional de Miller, con especial atención a los aspectos de diseño que minimizan el ruido y la tensión de offset del amplificador, en función de futuras aplicaciones en instrumentación ${ }^{[6]}$. 


\section{Metodología}

Para la etapa de diseño del AIO se propone un diagrama de flujo DDF básico para la toma y lectura de las señales mioeléctricas, iniciando con un solo canal, tomando el siguiendo DDF mostrado en la Fig.1.

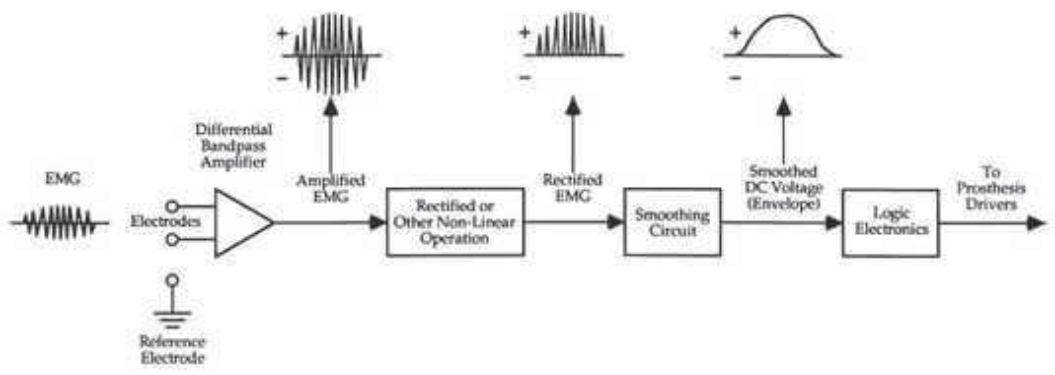

FigURA 1. DIAGRAMA DE FLUJO DE LA LECTURA Y CAPTURA DE SEÑALES MIOELECTRICAS

Con el DDF, se determinó sus distintos componentes a modelar e implementar en desarrollos a futuro, como son la etapa de preamplificación, amplificación, rectificación, filtrado y demás, las cuales deben ser controladas, diagnosticadas y visualizadas con apoyo en un software. El modelo desarrollado genera 3 subetapas en la trazabilidad del trabajo realizado a la señal mioeléctrica que se manifiesta en la Fig.2 ${ }^{[7],[8]}$.

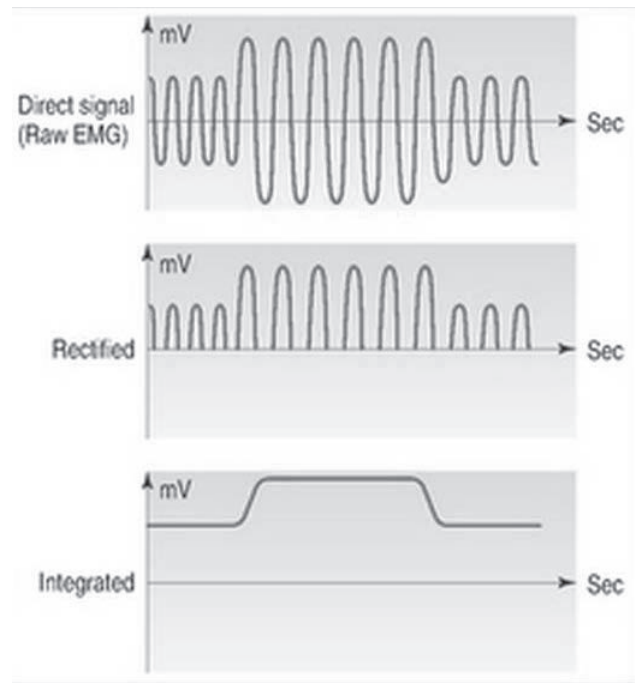

FIGURA 2. TRASABILIDAD DE LA DEPURACIÓN DE SEÑAL MIOELECTRICA 
Para el diseño y desarrollo del componente encargado de adquirir una señal a través de un sensor, se seleccionó el sensor HMC1501el cual se ajustó a través de un divisor de tensión y un operacional en configuración seguidor resistencia variable que cambia al aplicarle distintos valores en $U$ se puede obtener voltajes que varían entre oV y $2 \mathrm{~V}$ y los cuales pueden ser adquiridos por un conversor $A D C$ para su digitalización ${ }^{[9]}$. Su diseño esquemático se observa en la Fig.3.

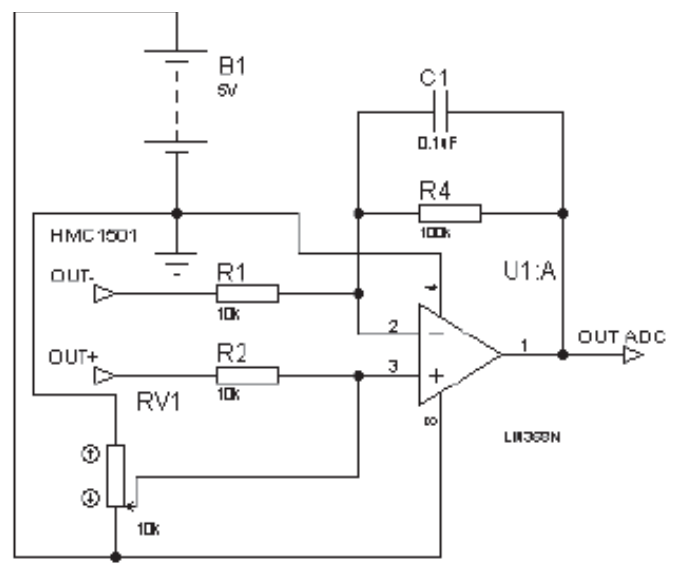

FIGURA 3. CIRCUITO CON FUNCIÓN DE SENSOR HMC1501

Ya determinado el sensor a ser utilizado en el diseño, se incorporó a continuación los sensores de fuerzas resistivos (FSR) las cuales tienen en su diagrama esquemático una resistencia variable que está en relación directa a la presión aplicada y se rige por el principio piezoresistivo. La sensibilidad al ruido/vibración no es alterada significativamente, los FSR trabajan con menor margen de error en superficies planas, fijas, estables y que estén lisas, su rango de sensibilidad de fuerza esta entre los $100 \mathrm{~g}$ hasta $10 \mathrm{Kg}$, y el rango de sensibilidad de presión obtenido esta entre los 1.5 psi hasta 150 psi. En la Fig.4. se observa el diagrama esquemático de un Circuito de señal tipo FSR 
Aguirre, et al. / Diseño y simulación de un amplificador "Instrumental Operacional"...

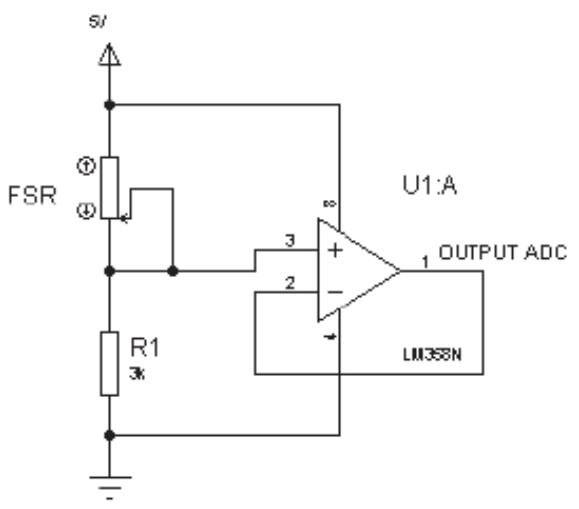

FIGURA 4. CIRCUITO SEÑAL FSR

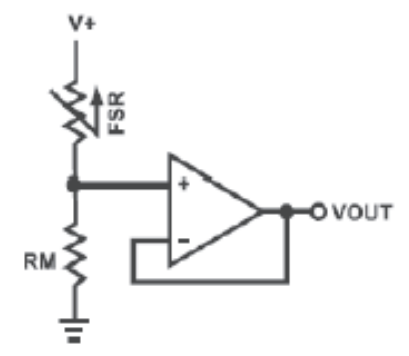

FIGURA 5. SENSOR DE FUERZA RESISTIVO FSR

Los elementos diferenciadores en el desarrollo del AOI es el de los filtros pre y pos amplificación,

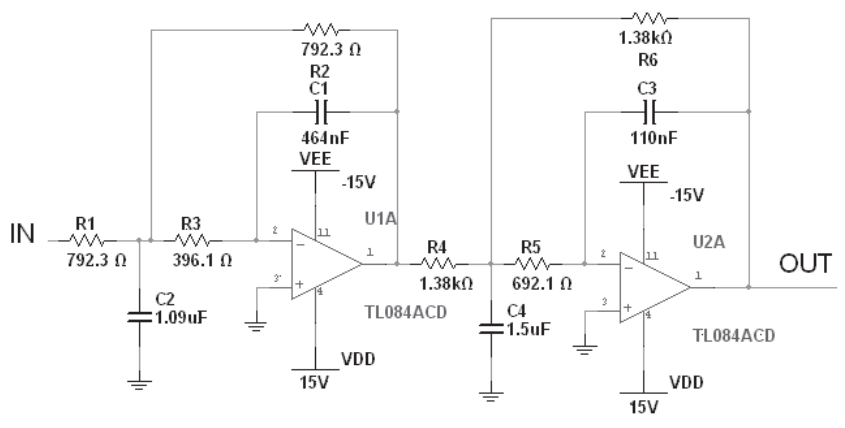

FIGURA 6. CIRCUITO FILTRO PASO BAJO 


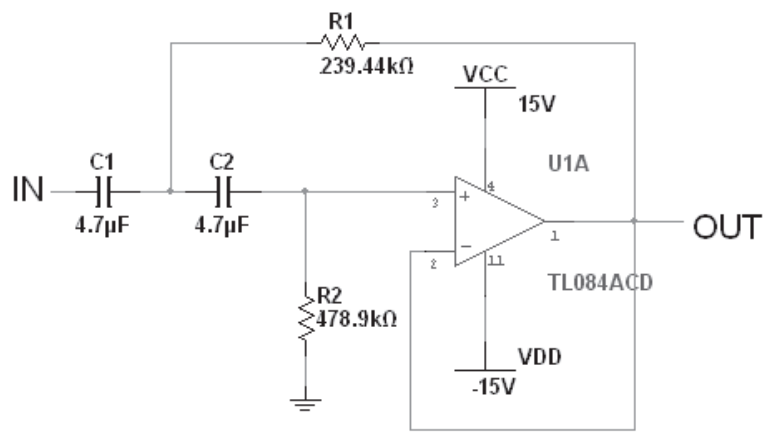

FiguRA 7. CIRCUITO FILTRO PASO ALTO

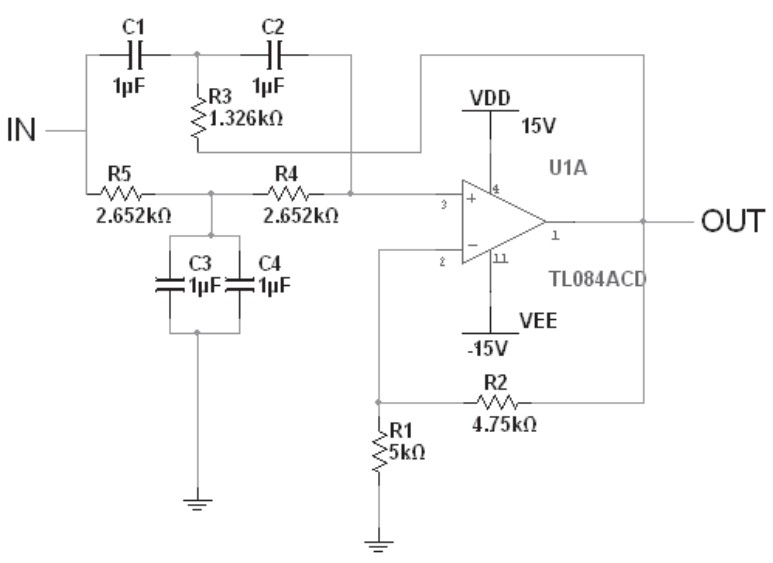

FIGURA 7. CIRCUITO FILTRO NOTCH

El estudio del amplificador que integre finalmente los elementos descritos con anterioridad se enfoca en dos modelos distintos, uno es, el amplificador tradicional basado en transistores, y el segundo modelo es el amplificador instrumental basado en el integrado TL084

Se estudian ambos amplificadores en paralelo para la elección de aquel que genera mayor beneficio en relación al control de ruido, filtro y ganancia de las señales mioeléctricas.

La Fig. 8. Muestra el esquema del segundo Amplificador 


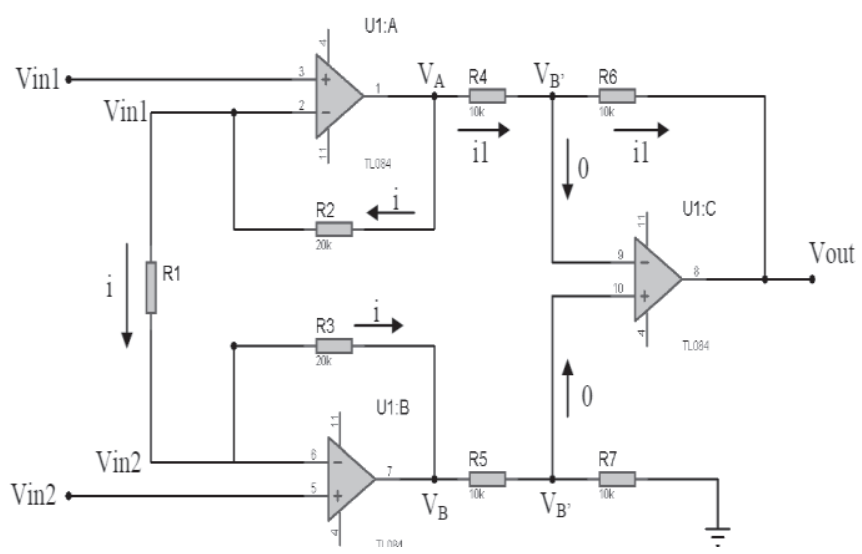

FIGURA 8. AMPLIFICADOR INSTRUMENTAL BASADO EN TL084

Se propone en un inicio seguir trabajando con una arquitectura tradicional de modelado del amplificador basado en transistores, el cual posee dos etapas que se observan en la Fig.9. Los transistores definidos como M1, M2, M3, M4 Y M5 son los que hacen parte de un amplificador diferencial de entrada considerando que los transistores definidos como M6 y M7 hacen parte de un amplificador inversor de salida. El valor de compensación en frecuencia se logra mediante el valor obtenido en los transistores M8 y CC. El M9, M10 y el M11 establecen la polarización de los transistores M5 y el M7.

Debido a que el ruido del amplificador es dominado por el ruido $1 / \mathrm{f}$ (flicker), se adopta una etapa de entrada PMOS por presentar menos ruido $1 / \mathrm{f}$ que los NMOS presentados por primera vez en la obra "Analog Integrated Circuit Design" por los autores Johns and Martín [2], lo que reduce y afecta negativamente otras características, entre ellas a la ganancia.

Gray and Meyer ${ }^{[3]}$ estudiaron el ruido y lo analizan por separado, tanto el ruido $1 / \mathrm{f}$ y el ruido denominado como ruido blanco, proponiendo lineamientos generales para la minimización de los mismos. La estrategia aplicada en el trabajo para la reducción del ruido 1/f consiste en disminuir la contribución de las cargas activas del diferencial de entrada se tiene, la que se logra adoptando longitudes de canal mayor para los transistores $\mathrm{M}_{3}$ y $\mathrm{M}_{4}$ respecto de las longitudes de los transistores M1 y M2. 
El control y lectura de señales mioeléctricas es probablemente el esquema de control más popular. Se basa en el concepto de que siempre que un músculo en el cuerpo se contrae o se flexiona, se produce una pequeña señal eléctrica (EMG) que es creada por la interacción química en el cuerpo. Esta señal es muy pequeña ( 5 a $20 \mu \mathrm{V}$ ) y se capta con electrodos superficiales que entran en contacto con la superficie de la piel.

Por lo anterior debemos hallar inicialmente la relación entre los transistores $L 1, L 3$ y $L 4$, y seria: $L 3=L 4=2 L 1$

Al observar que $L 1$ es la mitad de los otros, se determina que el ruido $1 / \mathrm{f}$ del amplificador queda dominado aproximadamente por la contribución del par diferencial (Transistores M1 y M2). Sabiendo que el ruido de un transistor depende en forma inversa de su área, dicha contribución puede ser reducida adicionalmente mediante un dimensionamiento adecuado, que se determina experimentalmente.

El método aplicado en el AIO para la reducción del ruido blanco es semejante al aplicado anteriormente, y se implementa disminuyendo las transconductancias de los transistores M3 y M4 frente a las de los transistores M1 y M2.

Por consiguiente, se obtiene que las relaciones de aspecto existente entre los transistores $\mathrm{M}_{1}$ y $\mathrm{M}_{2}$ son mucho mayores a las de los transistores $\mathrm{M}_{3}$ y M4. Aplicando por norma los valores $(\mathrm{W} / \mathrm{L}) 1=3 .(\mathrm{W} / \mathrm{L}) 3$

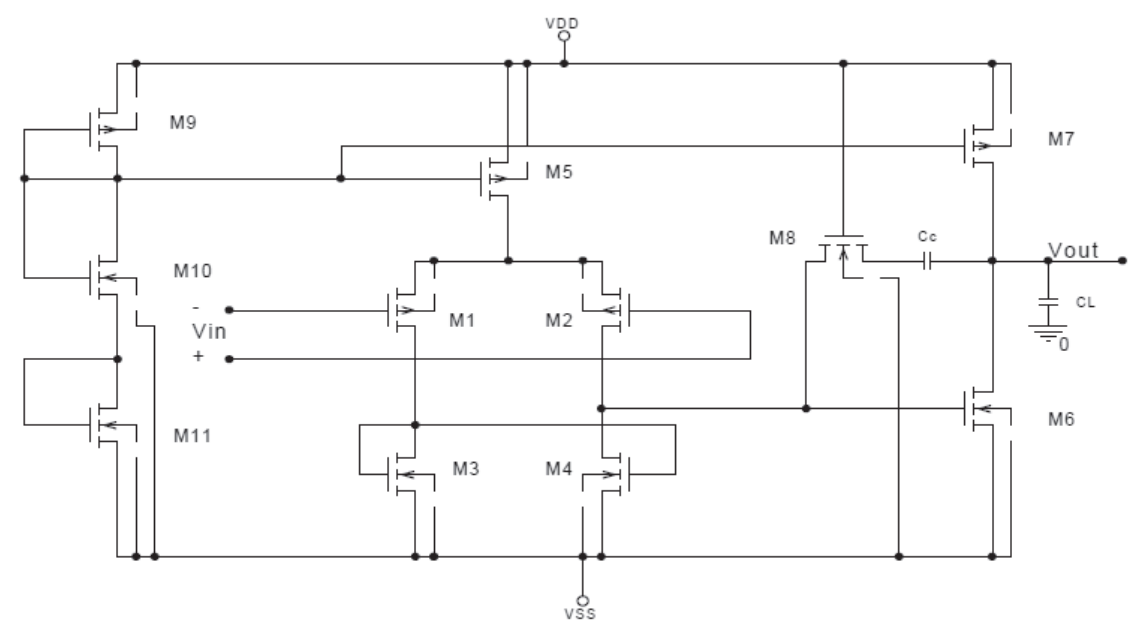

FIGURA 9. AMPLIFICADOR COMPUESTO POR TRANCISTORES 
Para disminuir el offset sistemático, se debe lograr que la tensión de reducción del transistor M4 sea la necesaria para que la corriente del transistor M6 sea semejante a la del transistor M7 bajo condiciones de entrada a cero. Para ello se establece, según lo propuesto por Johns, D and Martín, K. en su texto, Analog Integrated Circuit Design

$$
(\mathrm{W} / \mathrm{L}) 3 /(\mathrm{W} / \mathrm{L}) 6=(\mathrm{W} / \mathrm{L}) 4 /(\mathrm{W} / \mathrm{L}) 6=0.5(\mathrm{~W} / \mathrm{L}) 5 /(\mathrm{W} / \mathrm{L}) 7
$$

Por ende, las longitudes de los transistores $\mathrm{M}_{3}, \mathrm{M}_{4}$ y de $\mathrm{M}_{6}$ son iguales, con el propósito de asegurar que las relaciones anteriores se mantengan a pesar de variaciones inducidas por el proceso en las longitudes de canal de los transistores.

De acuerdo a lo tratado por Gregorian and Temes ${ }^{[4]}$. Analog MOS en su trabajo Integrated Circuits for Signal Processing, siendo tema de referencia en relación al offset aleatorio se reduce incrementando las transconductancias de los dispositivos de entrada ${ }^{[9]}$. Para esto se toman tensiones efectivas compuerta-surtidor de valor bajas para los transistores M1 y M2. Idénticamente, y con idéntico propósito (reducir offset), se implementan los transistores M5, M6 y M7 como la conexión en paralelo de transistores con W más pequeño [10],[11].

TABLA 1. PARÁMETROS GEOMÉTRICOS Y CORRIENTES DE POLARIZACIÓN. AlimentaCión $\pm 2,5$ voltios ${ }^{[12]}$

\begin{tabular}{|c|c|c|}
\hline Componente & W/L & Corriente $(\mu \mathrm{A})$ \\
\hline$M 1$ & $98 / 6$ & 10 \\
\hline$M 2$ & $98 / 6$ & 10 \\
\hline$M 3$ & $65.25 / 12$ & 10 \\
\hline$M 4$ & $65.25 / 12$ & 10 \\
\hline$M 5$ & $4 \times 25.5 / 6$ & 20 \\
\hline$M 6$ & $4 \times 65.25 / 12$ & 50 \\
\hline$M 7$ & $8 \times 25.5 / 5$ & 50 \\
\hline$M 8$ & $9.5 / 6$ & - \\
\hline$M 9$ & $25.5 / 6$ & 5 \\
\hline$M 10$ & $5 / 6$ & 5 \\
\hline$M 11$ & $9.75 / 6$ & 5 \\
\hline
\end{tabular}


La tabla 1 muestra los valores parámetros geométricos y las corrientes de polarización del amplificador diseñado con dicho fin al inicio, estos valores son determinados por los resultados obtenidos al realizar múltiples simulaciones con ellos.

\section{Resultados}

Los resultados que se presentan se obtienen mediante modelación en PROTEUS y en SPICE utilizando los modelos de los transistores provistos por el CNM. Y el amplificador operacional. Se utilizan los circuitos de prueba propuestos por Allen and Holberg [5]. CMOS en su obra titulada Analog Circuit Design para la caracterización de amplificadores operacionales, siendo la que menos ruido se generó al ser aplicada con múltiples valores de parámetros.

la respuesta en frecuencia (magnitud y fase) de la ganancia en modo diferencial que se obtiene con el AOI. Parametrizado es con una ganancia en DC de $84 \mathrm{~dB}$ con un producto ganancia ancho de banda (GBW) de $1.3 \mathrm{MHz}$ con una carga de 10pF. El lazo de compensación en frecuencia presenta un desempeño adecuado brindando un margen de fase de 56 grados.

La relación de rechazo de modo común es de $88 \mathrm{~dB}$ a frecuencias muy bajas.

En cuanto al rango dinámico de salida, las simulaciones muestran que el mismo es de 4.86 voltios.

La respuesta en gran señal se caracteriza mediante la medición de la velocidad de crecimiento (SR) y del tiempo del establecimiento (Ts) al 1\%. Para una carga capacitiva pura de $2 \mathrm{pF}$ se obtuvieron una SR de $1.6 \mathrm{v} / \mathrm{ms}$ y un Ts de $382 \mathrm{~ns}$, considerando que para 10pF los valores de SR y Ts fueron de $1.34 \mathrm{v} / \mathrm{ms}$ y 925 ns respectivamente. Estos son valores muy buenos para la velocidad de crecimiento (SR).

El voltaje de ruido de entrada equivalente $(\mathrm{nV} / \bullet \mathrm{Hz})$ encontrados en el modelo determinan que las contribuciones de los transistores $\mathrm{M}_{1}$ y $\mathrm{M}_{3}$ al ruido térmico de salida y al ruido $1 / f$ cumplen con las condiciones que se esperan desde la etapa de diseño y que son parte de los objetivos del diseño.

La tensión de offset del operacional se evalúa mediante simulación Montecarlo utilizando las desviaciones estadísticas del proceso brindadas por el 
Aguirre, et al. / Diseño y simulación de un amplificador "Instrumental Operacional"...

CNM. Se obtiene un offset referido a la entrada de 34.8 mvoltios bajo condición nominal con una desviación estándar (s) de 1.15 mvoltios.

\section{Conclusiones}

Se presenta en este trabajo el diseño de un amplificador operacional tanto Operacional como uno de tipo Miller con consideraciones de minimización de tensión de offset y de ruido, utilizando la tecnología CNM25 de $2.5 \mathrm{~mm}$. Obteniendo buenos resultados con el segundo tipo de amplificador

Las simulaciones muestran que el amplificador presenta una tensión de offset de entrada de 34.8mvoltios con una desviación estándar de 1.15 mvoltios.

En cuanto a características de ruido, se logra un voltaje de ruido de entrada equivalente de $55.44 \mathrm{nV} / \bullet \mathrm{Hz}, 26.75 \mathrm{nV} / \bullet \mathrm{Hz}$ y $24.5 \mathrm{nV} / \bullet \mathrm{Hz}$ para $10 \mathrm{~Hz}, 100$ $\mathrm{Hz}$ y $1 \mathrm{Khz}$ respectivamente. Los resultados cumplen con las condiciones de diseño al mostrar que los transistores del par diferencial de entrada dominan a los de la carga en cuanto a características de ruido flicker y ruido térmico.

Se logra así un amplificador con un desempeño adecuado en características de offset y ruido sin degradación elevada en sus otros parámetros ${ }^{[3]}$.

\section{Referencias}

[1] Laker, K and Sansen, W. Design of Analog Integrated Circuits and Systems. Mc. Graw Hill. 1994.

[2] Johns, D and Martín, K. Analog Integrated Circuit Design. John Wiley \& Sons. 1997.

[3] Gray, P and Meyer, R. "MOS Oparational Ampifier Design - A Tutorial Overview". Analog MOS Integrated Circuits II. IEEE Press. 1989.

[4] Gregorian, R and Temes, G. Analog MOS Integrated Circuits for Signal Processing. John Wiley \& Sons. 1986.

[5] Allen, P. and Holberg, D. CMOS Analog Circuit Design. Oxford University Press. 1987

[6] H. Poor, An Introduction to Signal Detection and Estimation. New York: Springer-Verlag, 1985 , ch. 4.

[7] J. U. Duncombe, "Infrared navigation—Part I: An assessment of feasibility (Periodical style)," IEEE Trans. Electron Devices, vol. ED-11, pp. 34-39, Jan. 1959.

[8] S. Chen, B. Mulgrew, and P. M. Grant, "A clustering technique for digital communications channel equalization using radial basis function networks," IEEE Trans. Neural Networks, vol. 4, pp. 570-578, July 1993. 
[9] R. W. Lucky, “Automatic equalization for digital communication,” Bell Syst. Tech. J., vol. 44, no. 4, pp. 547-588, Apr. 1965.

[10] E. H. Miller, "A note on reflector arrays (Periodical style-Accepted for publication)," IEEE Trans. Antennas Propagat., to be published.

[11] S. P. Bingulac, "On the compatibility of adaptive controllers (Published Conference Proceedings style)," in Proc. 4th Annu. Allerton Conf. Circuits and Systems Theory, New York, 1994, pp. 8-16.

[12] G. R. Faulhaber, "Design of service systems with priority reservation," in Conf. Rec. 1995 IEEE Int. Conf. Communications, pp. 3-8.

[13] W. D. Doyle, "Magnetization reversal in films with biaxial anisotropy," in 1987 Proc. INTERMAG Conf., pp. 2.2-1-2.2-6. 